\title{
An Infrared Luminous Merger with Two Bipolar Molecular Outflows : ALMA View of NGC 3256
}

\author{
K. Sakamoto ${ }^{1}$, S. Aalto ${ }^{2}$, F. Combes $^{3}$, A. Evans ${ }^{4,5}$ and A. Peck ${ }^{4}$ \\ ${ }^{1}$ ASIAA, Taiwan, ${ }^{2}$ Univ. Chalmers, OSO, Sweden, ${ }^{3}$ Observatoire de Paris, France, \\ ${ }^{4} \mathrm{NRAO}$, USA, ${ }^{5}$ Univ. Virginia, USA
}

\begin{abstract}
We found with ALMA 3D spectroscopy two bipolar molecular outflows in the luminous infrared galaxy NGC 3256. Each of the two merger nuclei has its own bipolar outflow.
\end{abstract}

Keywords. galaxies: active - galaxies: evolution - ISM: jets and outflows

Merging of disk galaxies tends to drive gas in the progenitor galaxies to their centers and thereby trigger intense star formation and/or nuclear activities there. The resulting high luminosity nuclei often have gas outflows, usually (but not necessarily always) perpendicular to the remnant disks. The already complex 3D structures of mergers are further complicated with this out-of-plane gas distribution and motion. It therefore needs high-quality data to recognize and characterize a luminous merger with its outflow(s).

Fig. 1 shows our recent ALMA observations of an infrared luminous merger NGC 3256, the most luminous galaxy within $\mathrm{z}=0.01$. In addition to the large-scale rotation with the major axis p.a. $\sim 75^{\circ}$ and velocity range within $V_{\text {sys }} \pm 200 \mathrm{~km} \mathrm{~s}^{-1}$ (left panel), there are high-velocity emission around the two nuclei (middle panel). The one around the southern merger nucleus is clearly bipolar and distinct from the large-scale gas motion (right panels). Subtracting it (in mind) in the middle panel, one can see another bipolar high-velocity gas around the northern nucleus. Our analysis suggests that the northern high-velocity gas is a starburst-driven molecular superwind with a wide opening angle and that the southern high-V gas is a well collimated bipolar molecular jet plausibly driven by an AGN in the southern nucleus (Sakamoto et al. 2014).

Spatially-resolved 3D studies like this are needed to accurately characterize the feedback processes in mergers through their evolutionary stages.
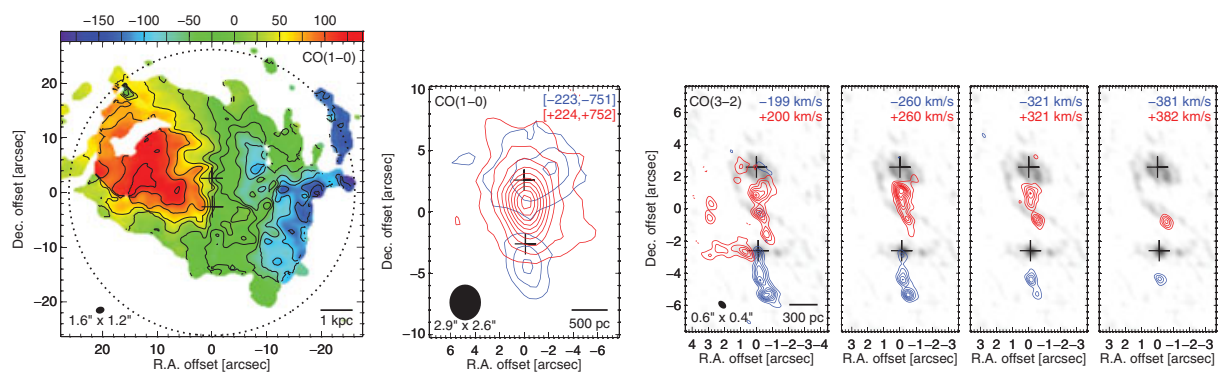

Figure 1. (Left) Overall $\mathrm{CO}$ velocity field. (Middle) $\mathrm{CO}(1-0)$ high-velocity gas. (Right 4 panels) $\mathrm{CO}(3-2)$ high-velocity gas. The two nuclei are marked with + signs.

\section{References}

Sakamoto, K., Aalto, S., Combes, F., Evans, A., \& Peck, A. 2014, submitted to ApJ 